\title{
Life-Threatening QT Prolongation in a Preterm Infant
}

\author{
Christian Paech, MD ${ }^{1}$ Roman Gebauer, MD ${ }^{1}$ Matthias Knüpfer, MD, PhD ${ }^{2}$ \\ ${ }^{1}$ Department for Pediatric Cardiology, University of Leipzig - Heart \\ Center, Leipzig, Germany \\ 2 Department of Neonatology, University of Leipzig, Leipzig, Germany \\ Address for correspondence Christian Paech, MD, Department for \\ Pediatric Cardiology, University of Leipzig - Heart Center, Strümpellstr. 39, \\ 04289 Leipzig, Germany (e-mail: christian.paech@med.uni-leipzig.de).
}

Am J Perinatol Rep 2014;4:e87-e88.
Abstract
Keywords
- long QT
- aqueductal stenosis
- hydrocephalus
- preterm

Introduction Temporary QT-interval prolongation following intracranial hemorrhage and hydrocephalus has been repeatedly reported in adults.

Case We report a case of excessive QT prolongation with sudden bradycardia resulting in 2:1 atrioventricular conduction in a preterm infant most likely associated with a congenital hydrocephalus. Pathomechanisms are discussed.

Conclusion Congenital hydrocephalus predisposes to excessive QT prolongation in preterm infants.
Temporary QT-interval prolongation following intracranial hemorrhage and hydrocephalus has been repeatedly reported in adults. ${ }^{1-3}$ To date, this association has never been reported in an infant.

\section{Case}

A preterm infant of 31 weeks' gestational age suffering from intrauterine aqueductal stenosis was referred to the neonatal intensive care unit after cesarean section for silent cervical opening. The aqueductal stenosis was diagnosed by prenatally performed magnetic resonance imaging. During the prenatal course, heart rate was within normal limits, without bradycardia or signs of heart block. Postnatal cardiopulmonary adaption was unremarkable and the basic heart rate was within normal limits for age. Cranial ultrasound after birth showed a hydrocephalus without signs of elevated intracranial pressure. On day 2 of life, the infant developed sudden bradycardia of $70 \mathrm{bpm}$ with poor peripheral perfusion and mild arterial hypotension, which resolved spontaneously after 10 minutes. Electrocardiogram (ECG) showed a sinus rhythm with excessive QT-interval prolongation (-Fig. 1). The resulting 2:1 atrioventricular (AV) conduction was due to ventricular refractoriness as reported in long QT syndrome (LQTS) patients, ${ }^{4}$ in which the atrial impulse is conducted via the AV node to the ventricular myocardium, but a QRS complex is missing because the ventricular tissue is still refractory due to the excessively prolonged repolarization period. Recognizably, this phenomenon is not an AV block on the basis of an impaired AV nodal function.

After initial variation of the QTc from 500 to 580 milliseconds with several episodes of bradycardia until day 5 of life, the QT interval then normalized continuously over 2 weeks. Repetitive ECGs performed throughout the hospital stay did not show any further QT prolongation. Despite the fact that there was no significant elevation of intracranial pressure during the first 2 weeks of life, a slow progressive rise was seen over the next 3 months and finally a ventriculo-peritoneal shunt had to be inserted. Family history of LQTS or sudden cardiac death was unremarkable and the identical twin brother had repetitive normal ECGs. Serum levels of potassium, calcium, and magnesium were within normal limits throughout the hospital stay. The patient did not receive any QT-prolonging drugs and episodes of QT prolongation were never noted again after initial normalization.

\section{Discussion}

Many factors, including electrolyte imbalances or hereditary LQTS, are reported to influence cardiac repolarization both in adult and pediatric patients. This case illustrates a life- received

April 9, 2014

accepted after revision

July 25, 2014

published online

November 10, 2014
DOI http://dx.doi.org/

10.1055/s-0034-1390166. ISSN 2157-6998.
Copyright $\odot 2014$ by Thieme Medical Publishers, Inc., 333 Seventh Avenue, New York, NY 10001, USA. Tel: +1(212) 584-4662.
License terms

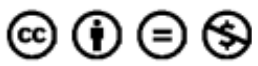




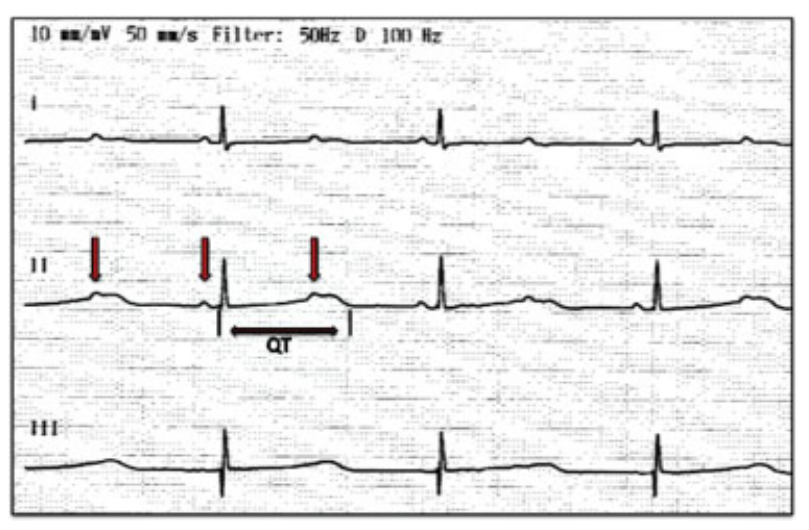

Fig. 1 An electrocardiogram on the 2 nd day of life is showing a sinus rhythm with $140 \mathrm{bpm}$ and an excessive QT prolongation (QTc $560 \mathrm{~ms}$ ) resulting in 2:1 atrioventricular (AV) conduction due to ventricular refractoriness. P-waves with normal AV conduction followed by a QRS complex and P-waves hidden in the ST-segment that are indicated (arrow).

threatening QT-interval prolongation associated with congenital hydrocephalus in a preterm infant. The incidence of QT-interval prolongation is reported with a range $11 \%$ to $66 \%$ in adults with hydrocephalus or intraventricular bleeding. ${ }^{2}$ The pathomechanism of these ECG changes is a manifestation of autonomic dysregulation, caused by overactivity of the sympathetic limb of the autonomic nervous system. ${ }^{3}$ As these findings have never been reported in a neonate before, it remains notional if the QT interval was influenced either by a hydrocephalus-causing impairment of the sympathetic-parasympathetic balance during the complex process of perinatal adaption or, for instance, by an intermittent cranial pressure elevation. Interestingly, the phenomenon of a dominant sympathetic activity in a preterm infant with hydrocephalus has already been reported by Uhrikova et al in 2012..$^{5}$ Thus, the lack of QT prolongation despite rising intracranial pressure over time and the exclusion of other reasons for a QT prolongation imply that an autonomic dysregulation might be causative in our patient. As the autonomic dysregulation is suspected to be a temporary phenomenon during the first days of life, that is, perinatal period, the disappearance of the QT prolongation after day 5 of life seems to suit this theory.

The combination of prematurity and postnatal adaption processes in the presence of congenital hydrocephalus seems predisposing. The authors suggest hemodynamic monitoring of these patients, as the reported phenomenon occurred suddenly, no data exist to identify affected individuals, and serious hemodynamic compromise has to be expected in case of a longer-lasting episode.

\section{Conclusion}

Congenital hydrocephalus may predispose to excessive QT prolongation in preterm infants.

\section{Author Contributions}

All authors have contributed to, read, and approved the manuscript.

\section{Conflict of Interest}

The authors have no conflicts of interest to disclose.

\section{References}

1 Qaqa AY, Suleiman A, Alsumrain M, Debari VA, Kirmani J, Shamoon FE. Electrocardiographic abnormalities in patients presenting with intracranial parenchymal haemorrhage. Acta Cardiol 2012; 67(6):635-639

2 van den Bergh WM, Algra A, Rinkel GJE. Electrocardiographic abnormalities and serum magnesium in patients with subarachnoid hemorrhage. Stroke 2004;35(3):644-648

3 van Bree MD, Roos YB, van der Bilt IAC, et al. Prevalence and characterization of ECG abnormalities after intracerebral hemorrhage. Neurocrit Care 2010;12(1):50-55

4 Cuneo BF, Etheridge SP, Horigome H, et al. Arrhythmia phenotype during fetal life suggests long-QT syndrome genotype: risk stratification of perinatal long-QT syndrome. Circ Arrhythm Electrophysiol 2013;6(5):946-951

5 Uhrikova Z, Kolarovszki B, Javorka K, et al. Changes in heart rate variability in a premature infant with hydrocephalus. AJP Rep 2012;2(1):43-46 